\title{
Use of Days of the Week in a Modified Mini-Mental State Exam (M-MMSE) for Detecting Geriatric Cognitive Impairment
}

\author{
Irene Hamrick, MD, Razia Hafiz, MD, and Doyle M. Cummings, PharmD
}

Purpose: The purpose of this study was to compare a modified version of the Mini-Mental State Examination (MMSE) with the standard MMSE and the Mini-Cog in patients $\geq 65$ years old, stratified by education and literacy level.

Method: This cross-sectional exploratory study enrolled a convenience sample of 219 patients with a complaint of memory loss or a diagnosis of dementia from a geriatric outpatient clinic, nursing home, senior center, and university hospital. The MMSE was administered, and in addition to spelling and serial $7 \mathrm{~s}$ backward, patients were asked to recite the days of the week backward with the intent to reduce educational bias. Scores on the modified MMSE were compared with scores of the MMSE and the MiniCog.

Results: Of the 219 patients, 157 were identified with cognitive impairment by the Mini-Cog. Using a cutoff of $\leq 23$, the MMSE identified 118 patients and the modified MMSE identified 91 patients with cognitive impairment, and with a cutoff of $\leq 27$ the MMSE identified 168 and the modified MMSE 149 patients. All cognitively intact subjects correctly recited the days of the week backward. Specificity of the modified MMSE was higher than the MMSE for most groups. The highest sensitivity and specificity (94\% and $88 \%$, respectively) as well as positive and negative predictive values ( $96 \%$ and $81 \%$, respectively) were in patients with low levels of education for the modified MMSE using a cut off of $\leq 27$.

Conclusion: Using the days of the week in the MMSE among illiterate and semiliterate participants and with education less than high school, and using a cutoff of 27 of 30, correlates better with Mini-Cog for dementia screening, with fewer false positives. (J Am Board Fam Med 2013;26:429-435.)

Keywords: Dementia, Elderly, Geriatrics, Memory, Nursing Homes, Screening

The US population is aging, ${ }^{1}$ and age is a risk factor for cognitive decline. The incidence of dementia doubles every 5 years after the age of 65 and

This article was externally peer reviewed.

Submitted 11 November 2012; revised 13 March 2013; accepted 25 March 2013.

From the Department of Family Medicine, University of Wisconsin, Madison (IH); Veterans Administration, Fayetteville, NC (RH); and the Department of Family Medicine, Brody School of Medicine, East Carolina University, Greenville, NC (DMC).

Funding: This study was supported by a Health Resources and Services Administration grant (1 K01 HP00159-01 Geriatric Academic Career Award (IH) that was awarded during the study. The Wooten Foundation provided \$3000 for a student to help with gathering of data for the study.

Prior presentation: This study was presented as a poster at the annual meeting of the American Geriatrics Society, Orlando, FL, May 12-15, 2010.

Conflict of interest: none declared.

Corresponding author: Irene Hamrick, MD, 1100 Delaplaine Ct., Madison, WI 53715 (E-mail: ihamrick@wisc.edu). reaches a prevalence of nearly $50 \%$ at age $85 .^{2}$ Yet dementia is not recognized by $67 \%$ of primary care providers. ${ }^{3}$ The Diagnostic and Statistical Manual of Mental Disorders (DSM-IV) criteria ${ }^{4}$ are used for diagnosis, but screening tests can identify patients at risk. Screening for the disease is important because of the long duration of the disease and high prevalence among the elderly. Health care providers have an opportunity to provide anticipatory guidance and help patients and families prepare for the financial burden of the disease and access community resources.

The Mini Mental State Examination (MMSE) is the most commonly used screening test ${ }^{5}$ and is useful in following disease progression over time. It has been used since 1975 as an integral instrument in screening for cognitive decline in the general population. ${ }^{6}$ The test evaluates cognitive level in 
several domains including reading, writing, and math. Its ability to accurately capture cognitive impairment is better in primary care than specialist settings. ${ }^{7}$ However, use MMSE has been questioned in subjects with lower levels of education, ${ }^{8-11}$ leading to adjustment of cut off points by education, with a cut off of 23 for high schooleducated patients and 27 for college- educated patients. ${ }^{9,12}$ Fillenbaum et $\mathrm{a}^{13}$ showed lower specificity for various screening tests in minorities, which correlated with education. False-positive results misclassify normal subjects as cognitively impaired and may generate unwarranted anxiety among patients and families. Artificially increased disease burden may affect research on health disparities and dementia. Several studies have sought to reduce the effect of educational bias in screening instruments for dementia. ${ }^{14-17}$ Attempts to improve the accuracy of screening have included adapting existing tests, adjusting cut-points and scores, establishing group norms, and replacing culture-specific items that are less dependent on language, literacy, and other skills developed during formal education..$^{8-11,14,18}$ The $3 \mathrm{MS}$ examination replaced reverse serial 7 counting with reverse 5 to 1 counting ${ }^{19}$ because of its difficulty. ${ }^{8}$ Tombaugh et $\mathrm{al}^{20}$ further adapted the $3 \mathrm{MS}$ examination by replacing reverse counting with reverse spelling. To our knowledge, no MMSE has been tested without a spelling subtest. Other tests that rely less on literacy and other skills related to formal education $^{18,21}$ include the 7 -minute screening battery ${ }^{17}$ the animal naming test ${ }^{22}$ and the time and change test. ${ }^{15}$ However, these tests do not lend themselves to monitoring disease progression over time and are not used as much as the MMSE.

Many of our patients with low levels of literacy, who could not spell or count backward, would often not continue the test. We have been piloting the use of saying the days of the week backward in place of counting or spelling backward, but review of the literature showed no studies validating its use, and only one study used an alternative that was not dependent on education. Blessed et $\mathrm{al}^{14}$ included months of the year backward in their screening tests for assessment of concentration. Its use was not validated nor evaluated for education or literacy. This article describes our preliminary evaluation of a modified version of the MMSE (MMMSE) with the addition of reciting the days of the week backward in the assessment of the con- centration and calculation domain of cognitive status in elderly patients with varying levels of education and literacy.

\section{Methods \\ Participants and Setting}

This study was designed as a cross-sectional comparison of cognitive assessment strategies. We enrolled a convenience sample of people $\geq 65$ years of age with memory complaints or a diagnosis of dementia who were seen in a geriatric outpatient center, family medicine clinic, and hospital of an academic medical system between January 2007 and April 2010; additional participants included residents of a nursing home and the local senior center.

Inclusion criteria were (1) age $\geq 65$ years; (2) English speaking; (3) informed consent to participate provided by the subject or subject's informant; and (4) subjective memory loss or cognitive decline reported by the patient or their informants or documented in the electronic medical record. Exclusion criteria include d (1) delirium or active psychosis; (2) severe language or physical disabilities that affect the reading, drawing, or written portions of the test or the hearing instructions; and (3) history of head trauma within the past 3 months.

Patients meeting the eligibility criteria were enrolled when they signed the informed consent. Subjects with limited or no literacy had the consent read to them and either signed or marked it themselves while witnessed by their informant(s) or signed by their informant(s). A copy of the signed document outlining the study objectives and risks and providing investigator contact information was given to the participants. Race was documented by the interviewer and cross-referenced in the medical record, where it was established by patient report. Patients or caregivers were asked about years of schooling and ability to read. When the patient or caregiver were unsure of literacy, the patient's ability to read a newspaper or medication insert was identified as literate and the ability read single words or simple commands was considered semiliterate. The institutional review board at East Carolina University approved our study.

\section{Interventions and Measures}

We used the Mini-Cog as control test for assessing cognition. This instrument was chosen because it 
has high sensitivity and specificity (99\% and 93\%, respectively) in patients with memory problems as identified by an interview with the patient's informant, similar to our population. ${ }^{23}$ Without arithmetic and spelling, the Mini-Cog is less affected by literacy or education. Its brevity fits into the busy schedule of a primary care practice. It consists of the 3-item recall, the same as in the MMSE, which was not repeated, and a clock drawing test. Patients were asked to draw the numbers and hands on an existing circle showing the time of 11:10 on the face of an analog clock. Scoring of the Mini-Cog starts with the 3-item recall. Patients who could remember and recall all 3 items were considered normal on the Mini-Cog; those who did not remember any items were identified as having cognitive impairment. The results of the clock drawing test determine the cognitive impairment of subjects scoring 1 or 2 points on the 3 -item recall. Those drawing the clock face with incorrect placement of or missing hands or numbers were classified as having cognitive impairment.

The Folstein MMSE was administered as recommended, using spelling world backward and counting backward by $7 \mathrm{~s}$ from 100 . We added a new question and asked patients to recite the days of the week backward (the M-MMSE) to test the potential of modifying the MMSE for people with low literacy and education.

The Folstein MMSE was purchased from Psychological Assessment Resources, Inc. (Lutz, FL) and administered by the authors and research assistants trained to administer screening tests; training of research assistants consisted of explanation of the test and supervision of at least 2 screening tests or until the principal investigator (IH) felt competency was achieved. The MMSE ${ }^{6}$ consists of answers that make up a total of 30 points. The concentration and calculation section of the standard version of the MMSE makes up 5 points: 1 point is scored for each correct serial subtraction of 7 from 100 up to a total of 5 consecutive instances (eg, 100, $93,86,79,72)$; 1 point is also scored for each letter of the word world recited backward correctly up to the total 5 points. The better of these separate results, either spelling or counting backward, is included in the total of the standard MMSE test. Any incorrect or out of sequence part was subtracted respectively (eg, "drlow" would have received 3 points). Patients then were asked to sequentially recite the days of the week backward, starting with any day; 1 point was given for each correct answer, up to a total of 5 points. The results of the days of the week in reverse were recorded in place of counting or spelling backward and calculated as the M-MMSE. The remaining items ${ }^{6}$ of the screening test were the same between the MMSE and the M-MMSE and were not repeated to avoid a training effect. Each person then had an MMSE score containing the better of the counting or spelling backward and an M-MMSE score containing the days of the week backward.

The Geriatric Depression Scale ${ }^{24}$ was administered if the MMSE score was $\leq 23$ to screen for depressive symptoms. Patients with a score of $>5$ (of 15) were given further evaluation, treatment, or both. These patients were not removed from the analysis; one meta-analysis shown that only $0.6 \%$ of dementia cases reversed with treatment of reversible causes. ${ }^{25}$

\section{Statistical Analysis}

Participants' total scores using the MMSE and the M-MMSE and the standard versus modified components were compared in reference to participant education level and standard MMSE cutoff scores. Scores also were compared by Mini-Cog classification rates of "normal" versus "cognitively impaired." Education was documented subjectively as recalled number of total years of schooling. For purposes of this analysis, participants were categorized by education levels of high school graduate and beyond ( $\geq 12$ years of schooling completed) and less than high school graduate $(<12$ completed years of schooling), with scores broken down into $\geq 24$ (not cognitively impaired, "normal") and $\leq 23$ (cognitively impaired). Demographic data detailing ethnicity, sex, and age were cross-tabulated with results. Descriptive statistics were used to characterize the study sample with respect to age, sex, and education/literacy status. Mean scores from each cognitive assessment strategy (MMSE, M-MMSE, Mini-Cog) were computed and compared for the overall group, as well as within subgroups by education and literacy status, using the Student $t$ test. Correlation between the M-MMSE and Mini-Cog was calculated with the Pearson coefficient. Using the Mini-Cog for comparison in identifying cognitive impairment, the sensitivity, specificity, and positive and negative predictive values of the MMSE and M-MMSE were compared using 2 different cut-points in the overall sample and in sub- 
Figure 1. Patient flow diagram.

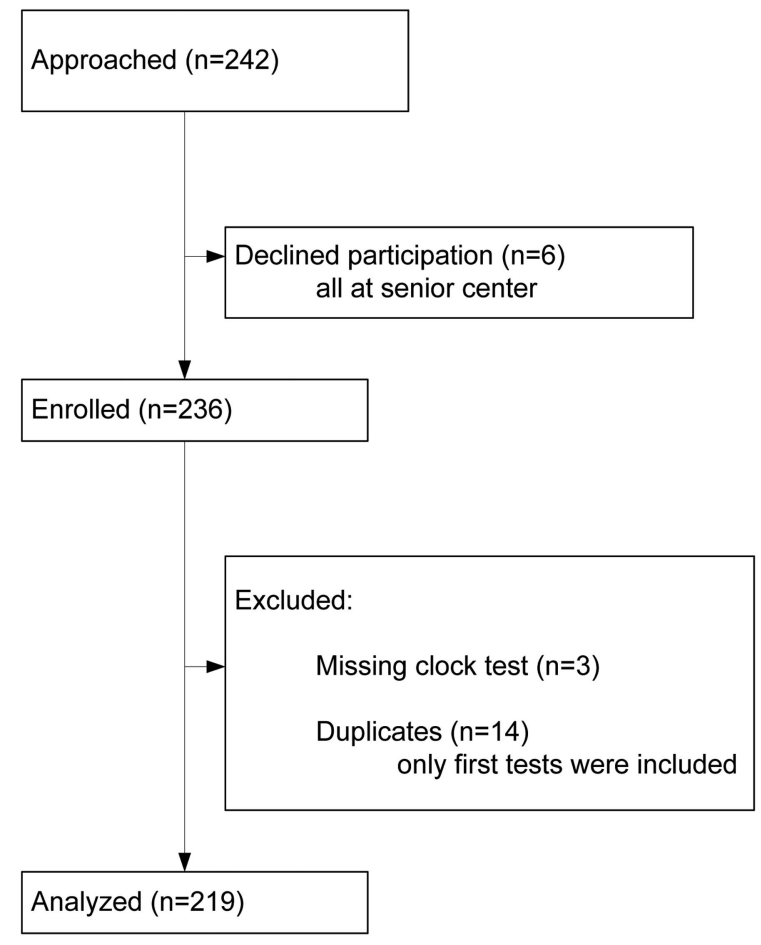

groups with limited education and literacy. While sensitivity and specificity establish validity, positive and negative predictive values describe utility in clinical settings. ${ }^{12}$ Statistical comparison of mean scores ( $t$ tests) was performed using SPSS version 16 (SPSS, Inc., Chicago, IL).

\section{Results}

This study enrolled 222 subjects with memory loss. Three did not perform the clock drawing test and were removed from the analysis (Figure 1). The mean \pm participant age was $80 \pm 7.0$ years (range, 64-93 years), and almost half ( $\mathrm{n}=108 ; 49.3 \%)$ of the participants graduated from high school. Most $(\mathrm{n}=145 ; 66.3 \%)$ described themselves as literate: 28 patients $(12.8 \%)$ were illiterate and $46(21.0 \%)$ were semiliterate. Almost half were African American ( $\mathrm{n}=107 ; 48.9 \%)$ and 157 (71.7\%) were classified with cognitive impairment through MiniCog scoring.

Average total scores achieved with the MMSE were lower than with the M-MMSE (Table 1) in subjects identified with cognitive impairment by Mini-Cog. This pattern was consistent regardless of literacy, education level, sex, or race (data not shown). Table 1 shows the results of the modified subcomponent (weekdays) versus the calculation (serial 7) or spelling (world) section analyzed separately, with up to 5 possible points each. All normal subjects correctly identified the days of the week backward, whereas $79 \%$ and $39 \%$ of normal subjects made mistakes on the counting and spelling backward, respectively.

The M-MMSE classified fewer patients with cognitive impairment than Mini-Cog or MMSE. Table 2 shows subjects identified with cognitive impairment by Mini-Cog, stratified by differing cutoff points for the respective standard or modified MMSE. Mini-Cog identified 157 patients with cognitive impairment, 86 of whom had less than a high school education, and 59 of whom reported being illiterate or semiliterate. Using a cutoff of 23, the M-MMSE identified fewer patients with cognitive impairment. This trend attenuates with decreased education and literacy. Using a cutoff of 27 for the MMSE, which is used for patients who are college graduates, the standard MMSE identified more patients with cognitive impairment than Mini-Cog, and the M-MMSE showed mixed results across education and literacy levels (Table 2).

Among patients considered normal by Mini$\operatorname{Cog}(n=62), 13$ patients scored $\leq 23$ by MMSE; of those, 4 had a high school education or more and 5 described themselves as literate. Using M-MMSE, 4 patients scored $\leq 23$; of those, 2 had a high school education or more and 2 described themselves as literate. Sensitivity was lower for the M-MMSE compared with MMSE; however, specificity and positive predictive value for the M-MMSE were higher in most subjects (Table 3).

By starting the concentration segment with the days of the week backward, we observed no abandoning of the screening process. Although we required all patients with an MMSE score of $\leq 23$ undergo depression screening, many more $(\mathrm{n}=$ 171) completed the Geriatric Depression Scale. Of those with a positive screen $(n=48 ; 28 \%)$, all were assured further evaluation and treatment.

The MMSE and M-MMSE were highly correlated with the Mini-Cog in patients with low levels of education and low literacy (Pearson coefficient, $0.51 ; P<.001)$. This study showed that a greater number of patients identified as normal by MiniCog are identified with cognitive impairment using the MMSE than the M-MMSE. This holds true for patients with low levels of education and low literacy. Conversely, a cutoff of $\leq 23$ (of 30 ) on 
Table 1. Mean Score on Tests and Subtests by Cognitive Status as Determined by Mini-Cog

\begin{tabular}{|c|c|c|c|}
\hline Test/Subtest (All Subjects) & Cognitive Impairment $(\mathrm{n}=157)$ & Normal $(\mathrm{n}=62)$ & $P$ \\
\hline MMSE & $19.39(5.48)$ & $26.03(3.28)$ & $<.001$ \\
\hline M-MMSE & $20.88(5.53)$ & $27.03(2.25)$ & $<.001$ \\
\hline Serial 7s & $0.90(1.52)$ & $2.02(1.87)$ & $<.01$ \\
\hline Spelling & $2.24(2.03)$ & $3.85(1.69)$ & $<.001$ \\
\hline Weekdays & $3.88(1.91)$ & $5.00(0)$ & $<.001$ \\
\hline High school graduate & Cognitive Impairment $(\mathrm{n}=71)$ & Normal $(\mathrm{n}=37)$ & \\
\hline MMSE & $21.62(4.63)$ & $26.78(2.69)$ & $<.001$ \\
\hline M-MMSE & $22.69(4.67)$ & $27.27(2.17)$ & $<.001$ \\
\hline Less than high school & Cognitive Impairment $(\mathrm{n}=86)$ & Normal $(\mathrm{n}=25)$ & \\
\hline MMSE & $17.56(5.47)$ & $24.92(3.80)$ & $<.001$ \\
\hline M-MMSE & $19.38(5.75)$ & $26.68(2.36)$ & $<.001$ \\
\hline Literate & Cognitive Impairment $(\mathrm{n}=98)$ & Normal $(\mathrm{n}=47)$ & \\
\hline MMSE & $20.59(5.31)$ & $26.91(2.55)$ & $<.001$ \\
\hline M-MMSE & $21.74(5.49)$ & $27.36(2.05)$ & $<.001$ \\
\hline Illiterate and semiliterate & Cognitive Impairment $(\mathrm{n}=59)$ & Normal $(\mathrm{n}=15)$ & \\
\hline MMSE & $17.31(5.21)$ & $23.27(3.85)$ & $<.001$ \\
\hline M-MMSE & $19.38(5.36)$ & $26.00(2.59)$ & $<.001$ \\
\hline
\end{tabular}

Values are means (standard deviations). Maximum possible scores for weekdays, serial 7, or spelling were 5 and for Mini-Mental State Examination (MMSE) or Modified-MMSE (M-MMSE) were 30. For all tests, normal patients had a significantly higher mean score than patients with cognitive impairment, including those with less than a high school education or who were illiterate or semiliterate. The M-MMSE showed results in the normal range for normal subjects more frequently (higher score). Although the score for patients identified with cognitive impairment also was higher, it still fell in the abnormal range. All normal subjects correctly recited the days of the week backward.

the MMSE may falsely identify many patients with the disease. Moving the cutoff to $\leq 27$ and using the M-MMSE may be a more accurate cut

Table 2. Number and Subsets of Patients With Cognitive Impairment as Identified by the Mini-Mental State Examination (MMSE), the Modified MSME (M-MMSE), and the Mini-Cog*

\begin{tabular}{lcc}
\hline & \multicolumn{2}{c}{ Cutoff Scores } \\
\cline { 2 - 3 } & $\begin{array}{c}\text { MMSE } \\
\leq 23\end{array}$ & $\begin{array}{c}\text { MMSE } \\
\leq 27\end{array}$ \\
Test by Subset of Patients & & \\
\hline All patients $(\mathrm{n}=219)$ & 157 & 157 \\
$\quad$ Mini-Cog & 118 & 168 \\
MMSE & 91 & 149 \\
M-MMSE & & \\
Patients with <high school education & & \\
$\quad(\mathrm{n}=111)$ & 86 & 86 \\
Mini-Cog & 83 & 88 \\
MMSE & 53 & 83 \\
M-MMSE & & \\
Illiterate or semiliterate patients $(\mathrm{n}=74)$ & 59 & 59 \\
Mini-Cog & 59 & 70 \\
MMSE & 46 & 65 \\
M-MMSE & & \\
\hline
\end{tabular}

*Using cutoffs of 23 and 27 points from possible 30 respectively. off for identifying patients with low levels of education with cognitive impairment. Therefore, we calculated the specificity and sensitivity, shown in Table 3. Sensitivity of the M-MMSE is generally lower compared with the MMSE, with higher specificity. Positive predictive values are generally better for the M-MMSE than the MMSE, except for the M-MMSE at a cutoff of 27 in all subjects. The highest predictive value is in patients with low levels of education using the M-MMSE at a cutoff of 27 .

\section{Discussion}

To our knowledge this is the first study to test a version of the MMSE that is less dependent on spelling or calculation in subjects with low literacy and levels of education. We found that among illiterate and semiliterate participants and those with less than a high school education, the MMMSE (using a cutoff of 27 of 30) correlates better with Mini-Cog for cognitive impairment screening, with fewer false positives. Our efforts were focused on developing a method of accurate dementia screening for populations in the counties of Eastern North Carolina, where proportion estimates of 
Table 3. Sensitivity/Specificity and Positive/Negative Predictive Values of 2 Cutoff Scores for the Mini Mental State Examination (MMSE) and Modified MMSE (M-MMSE) Using the Mini-Cog to Identify Cognitive Impairment

\begin{tabular}{|c|c|c|c|c|}
\hline & \multicolumn{4}{|c|}{ Cutoff Scores } \\
\hline & MMSE $<23$ & M-MMSE $<23$ & MMSE <27 & M-MMSE $<27$ \\
\hline \multicolumn{5}{|l|}{ Sensitivity/specificity } \\
\hline All subjects $(\mathrm{n}=219)$ & $0.72 / 0.77$ & $0.61 / 0.93$ & $0.73 / 0.77$ & $0.89 / 0.53$ \\
\hline Education $<12$ th grade $(\mathrm{n}=111)$ & $0.84 / 0.60$ & $0.73 / 0.92$ & $0.96 / 0.32$ & $0.94 / 0.88$ \\
\hline Low literacy $(\mathrm{n}=74)$ & $0.84 / 0.40$ & $0.74 / 0.87$ & $0.97 / 0.13$ & $0.93 / 0.33$ \\
\hline \multicolumn{5}{|l|}{ Positive/negative predictive values } \\
\hline All subjects $(\mathrm{n}=219)$ & $0.89 / 0.53$ & $0.96 / 0.49$ & $0.89 / 0.53$ & $0.82 / 0.66$ \\
\hline Education $<12$ th grade $(\mathrm{n}=111)$ & $0.88 / 0.52$ & $0.97 / 0.50$ & $0.83 / 0.73$ & $0.96 / 0.81$ \\
\hline Low literacy $(\mathrm{n}=74)$ & $0.84 / 0.40$ & $0.96 / 0.46$ & $0.81 / 0.50$ & $0.84 / 0.56$ \\
\hline
\end{tabular}

level I literacy, corresponding to 0 to 8 years of schooling and the lowest level established by the National Adult Literacy Survey, can reach over $27 \%{ }^{26}$ A third of the participants of a $1993 \mathrm{Na}$ tional Adult Literacy Survey who performed at level I of the 3 levels of the survey were $\geq 65$ years old. ${ }^{27}$ These numbers are even higher in African Americans, who comprise almost a third of the region's population ${ }^{28}$ and about half of our clinic population.

We expected the false positive rate for the MMMSE to be lower than for the MMSE, considering that all normal subjects correctly recited the days of the week backward. However, only the low literacy group showed a lower false positive rate in the M-MMSE.

Strengths of our study include the comparison with the Mini-Cog, a validated test, and the variety of settings that included a hospital, outpatient clinic, nursing home, and senior center, making this a representative sample with broad applicability. Other strengths are an almost equal number of minority subjects and relatively good representation of subjects with low literacy and levels of education. Data regarding dementia among minority populations are largely absent, and the evaluation of screening instruments such as the MMSE were mostly based on white populations with an underrepresentation of minorities in Alzheimer disease research. ${ }^{29,30}$

Limitations of our study include a convenience sample that may not be representative of the general population. Education level defined by years of schooling was subject to recall bias, with no documentation or quality measure. Literacy, which is inherently difficult to measure, was similarly identified by subjective patient report of the ability to read a newspaper and/or simple directions. Some patients with normal Mini-Cog had abnormal MMSE and vice versa. While the diagnosis of dementia is based on the DSM-IV criteria ${ }^{4}$ and depends on decline over time, this could not be formally done in this crosssectional pilot study. This study provides some evidence that the days of the week could be used in place of spelling or counting backward in the MMSE. With the current limitations, the efficacy of this approach cannot be shown conclusively. Further studies testing the M-MMSE using DSM-IV criteria are needed.

\section{Conclusion}

Our study shows that this modified screening instrument may lead to fewer participants being incorrectly categorized as cognitively impaired solely due to low literacy level. Taking the educational part (spelling and counting) out of the MMSE, using days of the week recited backward as the Modified-MMSE, and using a cut off 27 of 30 for low literacy, the M-MMSE detects cognitive impairment with fewer false positives.

The authors thank Andreea Doaga, MD, for her role in the inception and early conduct of the study; Hossein Movahed, $\mathrm{MD}$, for help with the analysis; Charles Baker, MPH, for his contributions to previous versions of the manuscript; and Mary Beth Plane, $\mathrm{PhD}$, for review of the manuscript.

\section{References}

1. Administration on Aging, Department of Health and Human Services. Aging statistics. Last modified May 8, 2013. Available from: http://www.aoa.gov/AoA Root/Aging_Statistics/index.aspx. Accessed May 17, 2013.

2. Mohs RC, Breitner JC, Silverman JM, Davis KL. Alzheimer's disease. morbid risk among first-degree relatives approximates $50 \%$ by 90 years of age. Arch Gen Psychiatry 1987;44:405-8. 
3. Valcour VG, Masaki KH, Curb JD, Blanchette PL. The detection of dementia in the primary care setting. Arch Intern Med 2000;160:2964-8.

4. American Psychiatric Association, American Psychiatric Association. Task Force on DSM-IV. Diagnostic and statistical manual of mental disorders. 4th ed. Washington, DC: American Psychiatric Association; 1994.

5. Shulman KI, Herrmann N, Brodaty H, et al. IPA survey of brief cognitive screening instruments. Int Psychogeriatr 2006;18:281-94.

6. Folstein MF, Folstein SE, McHugh PR. "Mini-mental state". A practical method for grading the cognitive state of patients for the clinician. J Psychiatr Res 1975;12:189-98.

7. Mitchell AJ. A meta-analysis of the accuracy of the mini-mental state examination in the detection of dementia and mild cognitive impairment. J Psychiatr Res 2009;43:411-31.

8. Anthony JC, LeResche L, Niaz U, von Korff MR, Folstein MF. Limits of the 'Mini-Mental State' as a screening test for dementia and delirium among hospital patients. Psychol Med 1982;12:397-408.

9. Crum RM, Anthony JC, Bassett SS, Folstein MF. Population-based norms for the Mini-Mental State Examination by age and educational level. JAMA 1993;269:2386-91.

10. Bassett SS, Folstein MF. Memory complaint, memory performance, and psychiatric diagnosis: a community study. J Geriatr Psychiatry Neurol 1993;6:105-11.

11. Wood RY, Giuliano KK, Bignell CU, Pritham WW. Assessing cognitive ability in research: use of MMSE with minority populations and elderly adults with low education levels. J Gerontol Nurs 2006;32:45-54.

12. O'Bryant SE, Humphreys JD, Smith GE, et al. Detecting dementia with the mini-mental state examination in highly educated individuals. Arch Neurol 2008;65:963-7.

13. Fillenbaum G, Heyman A, Williams K, Prosnitz B, Burchett B. Sensitivity and specificity of standardized screens of cognitive impairment and dementia among elderly black and white community residents. J Clin Epidemiol 1990;43:651-60.

14. Blessed G, Tomlinson BE, Roth $M$. The association between quantitative measures of dementia and of senile change in the cerebral grey matter of elderly subjects. Br J Psychiatry 1968;114:797-811.

15. Inouye SK, Robison JT, Froehlich TE, Richardson ED. The time and change test: a simple screening test for dementia. J Gerontol A Biol Sci Med Sci 1998;53:M281-6.

16. Willis SL, Allen-Burge R, Dolan MM, Bertrand RM, Yesavage J, Taylor JL. Everyday problem solv- ing among individuals with Alzheimer's disease. Gerontologist 1998;38:569-577.

17. Solomon PR, Pendlebury WW. Recognition of Alzheimer's disease: the 7 Minute Screen. Fam Med 1998;30:265-71.

18. Borson S, Scanlan JM, Chen P, Ganguli M. The Mini$\mathrm{Cog}$ as a screen for dementia: validation in a population-based sample. J Am Geriatr Soc 2003;51:1451-4.

19. Teng EL, Chui HC. The Modified Mini-Mental State (3MS) examination. J Clin Psychiatry 1987;48:314-8.

20. Tombaugh TN, Mcdowell I, Kristjansson B, Hubley AM. Mini-Mental State Examination (MMSE) and the Modified MMSE (3MS): a psychometric comparison and normative data. Psychol Assess 1996;8:48-59.

21. Borson S, Brush M, Gil E, et al. The Clock Drawing Test: utility for dementia detection in multiethnic elders. J Gerontol A Biol Sci Med Sci 1999;54: M534-40.

22. Tombaugh TN, Kozak J, Rees L. Normative data stratified by age and education for two measures of verbal fluency: FAS and animal naming. Arch Clin Neuropsychol 1999;14:167-77.

23. Borson S, Scanlan J, Brush M, Vitaliano P, Dokmak A. The Mini-Cog: a cognitive 'vital signs' measure for dementia screening in multi-lingual elderly. Int J Geriatr Psychiatry 2000;15:1021-7.

24. Sheikh JI, Yesavage JA, Brooks JO 3rd, et al. Proposed factor structure of the Geriatric Depression Scale. Int Psychogeriatr 1991;3:23-8.

25. Clarfield AM. The decreasing prevalence of reversible dementias: an updated meta-analysis. Arch Intern Med 2003;163:2219-29.

26. National Center for Education Statistics. National Assessement of Adult Literacy. http://nces.ed.gov/naal/ estimates/stateestimates.aspx Accessed June 18, 2013.

27. Kirsch IS, U.S. Office of Educational Research and Improvement, Educational Testing Service, $\mathrm{Na}$ tional Center for Education Statistics. Adult literacy in America: a first look at the results of the National Adult Literacy Survey. 2nd ed. Washington, DC: Office of Educational Research and Improvement, U.S. Department of Education; 1993.

28. East Carolina University. Eastern North Carolina Dataset Project. Available from: http://core.ecu.edu/ umc/enc/index.htm. Accessed March 21, 2012.

29. Connell CM, Shaw BA, Holmes SB, Foster NL. Caregivers' attitudes toward their family members' participation in Alzheimer disease research: implications for recruitment and retention. Alzheimer Dis Assoc Disord 2001;15:137-45.

30. Holston EC. Stigmatization in Alzheimer's disease research on African American elders. Issues Ment Health Nurs 2005;26:1103-27. 